\title{
Acoustic Streaming Visualization in Elastic Spherical Cavities
}

\author{
Sznitman, J.*1,2 and Rösgen, T.*1 \\ *1 Institute of Fluid Dynamics, ETH Zurich, CH-8092 Zurich, Switzerland. \\ *2 Present address: Department of Mechanical Engineering \& Applied Mechanics, University of \\ Pennsylvania, Philadelphia, PA 19104 USA. E-mail: joss@seas.upenn.edu \\ Received 28 November 2007 \\ Revised 27 May 2008
}

\begin{abstract}
Flow visualizations are presented for acoustic streaming occurring inside spherical elastic cavities oscillating in an acoustic field. Streaming flows are visualized using Particle Image Velocimetry (PIV) and results are observed for a range of values of a dimensionless frequency parameter, $M=120-306$. Over the frequency range investigated, streaming flow fields remain steady at a given value of $M$. The magnitude of the flows circulating inside the cavity remains small $(<1 \mathrm{~mm} / \mathrm{s})$ and follows a non-linear dependency with respect to the acoustic power of the sound wave. The present boundary-driven cavity flows may enhance particle fluid transport mechanisms, leading ultimately to potential fluid mixing applications.
\end{abstract}

Keywords : Acoustic streaming, Cavity flow, Low Reynolds number flow, PIV.

\section{Introduction}

It is well-known that the propagation of sound waves in a fluid may lead to a bulk non-periodic motion of the fluid. This nonlinear phenomenon is called acoustic streaming and has been reviewed extensively (Lighthill, 1978; Riley, 2001). In particular, a theory for streaming flows around oscillating bodies has been developed for gas bubbles undergoing harmonic translating and volume oscillation modes in a liquid. Davidson and Riley (1971) considered small-scale acoustic streaming (microstreaming) in a liquid outside an isolated gas bubble induced by the translational harmonic oscillation of the bubble. More recently, $\mathrm{Wu}$ and $\mathrm{Du}$ (1997) extended this work by studying the streaming behavior of a gas bubble simultaneously undergoing translating oscillations and volume oscillations. In parallel, theoretical descriptions of acoustic streaming occurring near a fluid-solid interface, rather than a fluid-gas one, have been developed (Carlsson et al., 2004), giving rise to flow patterns outside solid spheres similar to ones observed near gas bubbles (Lee and Wang, 1990).

Although acoustic streaming has been studied for over half a century, there exists nevertheless limited flow visualization data available. Experimentally, streaming flows are difficult to evaluate because of their small velocities and displacements. Kolb and Nyborg (1956) were the first to observe vortex motions in the vicinity of small vibrating sources in liquids. Since then, qualitative (Gormley and $\mathrm{Wu}, 1998$ ) and recent quantitative studies (Kotas et al., 2007; Tho et al., 2007) have completed the literature. While evidence of investigations pertaining to acoustic streaming occurring inside a cavity may be found ( $\mathrm{Oh}$ and Yang, 2005), the bulk of studies have rather concentrated on resolving streaming flows occurring outside an oscillating cavity. This has been in part motivated by bio-engineering applications, such as soluminescence, ultrasound contrast agents (Gormley and Wu, 1998), and sonoporation as a result of the shearing action of 
microstreaming flows (Wu et al., 2002). In particular, it has been experimentally shown that streaming in a fluid outside a gas bubble may be used for microfluidic applications, such as in the transport of particles (Marmottant and Hilgenfeldt, 2004) or in the mixing of fluids at the microscale (Liu et al., 2002).

While there exist theoretical investigations for streaming flows generated inside an oscillating gas bubble (Wu et al., 1997) or a levitated drop (Zhao et al., 1999), there is however to our knowledge, few if any experimental visualizations of internal streaming flows generated at a solid-fluid interface. One reason may come from the difficulty of choosing an appropriate experimental setup illustrating internal streaming flows generated at a solid-fluid boundary, while simultaneously allowing sufficient optical access for measurement techniques. Therefore, in an effort to complete existing flow-visualization studies on the topic, we have investigated acoustic streaming inside transparent, air-filled, thin elastic spherical cavities. Our experimental approach is based on particle image velocimetry (PIV) measurements inside elastic spherical cavities. Our results suggest that multiple modes of membrane oscillations exist, leading to a variety of complex acoustic streaming flow patterns. It is our belief that these boundary-driven cavity flows may enhance the transporting mechanisms of a fluid inside the elastic chamber, leading to potential applications in (micro-)mixing, similar to designs involving peristatically induced flow motion in closed micro-cavities with vibrating walls (Yi et al., 2002).

(a)

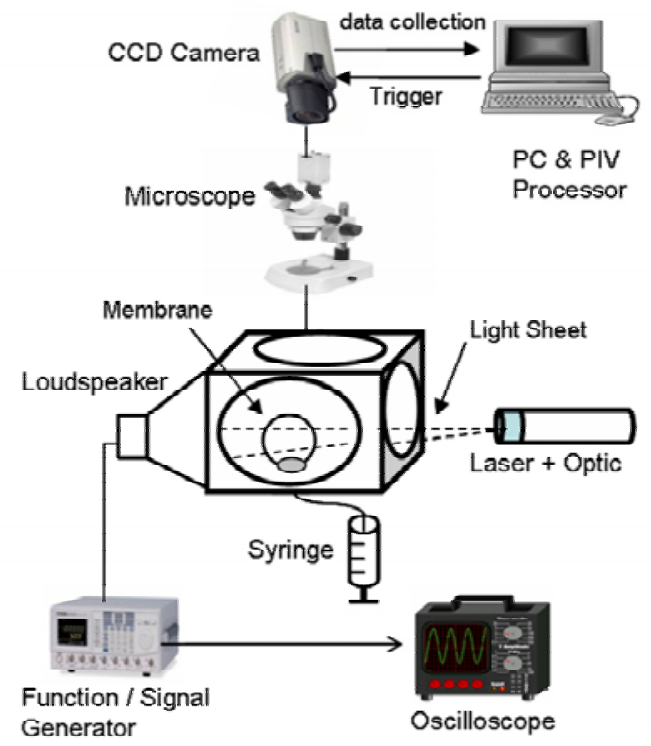

(b)

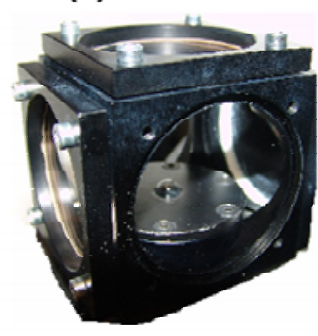

(c)

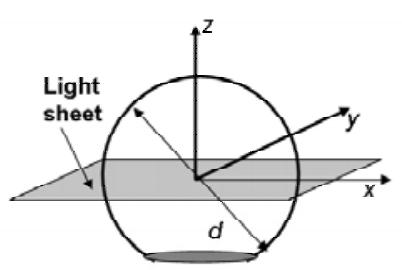

Fig. 1. (a) Schematic of the experimental apparatus, (b) Close-up of the test cell with an inflated membrane, (c) View of measurement plane inside the cavity, including coordinate system.

\section{Experimental Methods and Procedures}

\subsection{Apparatus}

The experimental apparatus (Fig. 1(a)) consists of a test cell enclosing an inflated elastic membrane, a loudspeaker and an imaging system. The test cell is made of a cubic-shaped $\left(\sim 5 \mathrm{x} 5 \mathrm{x} 5 \mathrm{~cm}^{3}\right)$ aluminum frame with detachable cover plates, each of which may be mounted on five of the faces of the cube (four side faces and the top face). Each plate guarantees hermetical sealing of the test volume. Side plates may either be painted black to minimize scattering of laser light, or designed with a circular glass window for optical access into the chamber (Fig. 1(b)). The remaining bottom face of the cube is fitted with a thin transparent silicone elastomer film (50 $\mu \mathrm{m}$ thick, $\rho=1260$ 
$\mathrm{kg} / \mathrm{m}^{3}$, Goodfellow Cambridge Limited, UK) sandwiched between a bottom cylindrical slab and an exchangeable upper plate designed with different circular orifice opening sizes with a rounded edge. In this way, the silicone membrane is tightly clamped between both plates and a silicone paste is first applied at the contact between the elastic membrane and the bottom slab to guarantee negligible air leakage upon inflation. The bottom slab is drilled with a central hole connected to a graduated syringe. In turn, thin elastic bubbles may be inflated by injecting air through the orifice opening. Typical elastic cavities are generated at a $6 \mathrm{~mm}$ circular orifice opening, by inflating $\sim 1.5-2$ $\mathrm{ml}$ of air, resulting in a spherical cap with a characteristic diameter of $d \sim 6.5-7 \mathrm{~mm}$. A schematic of the measurement plane is illustrated in Fig. 1(c).

Acoustic excitation is generated with a piezoelectric loudspeaker mounted onto one of the test cell faces and connected to a signal generator which delivers a sinusoidal electrical waveform. The waveform frequency may be varied from 0 to $20 \mathrm{kHz}$ in increments of $\sim 0.01 \mathrm{kHz}$ and the voltage amplitude (peak-to-peak, $V_{p p}$ ) between 3 and $20 V_{p p}$ in increments of $0.1 \mathrm{~V}$. These input parameters are verified by an oscilloscope (Agilent 54622A MegaZoom) connected to the signal generator. Sound pressure levels emitted by the loudspeaker vary nonlinearly with its excitation frequency. To determine the actual acoustic power delivered inside the chamber, the power $(\mathrm{dB})$ was measured as a function of the input frequency, $f$, and voltage amplitude, $V_{p p}$. These were recorded using a sound level meter (Voltcraft SL-100) with a resolution of $0.1 \mathrm{~dB}$ and accuracy of $\pm 2 \mathrm{~dB}$. The microphone head was placed directly above the orifice opening, at a distance of $\sim 25 \mathrm{~mm}$ away from the loudspeaker. Results are plotted in Fig. 2 over the principal range of frequencies investigated.

The imaging system consists of a progressive scan CCD camera (Pulnix TM) with $15 \mathrm{~Hz}$ image acquisition rate and a resolution of $1008 \times 1008$ pixels triggered via computer control. The camera is fitted onto a microscope such that typical inflated cavities are imaged with a field of view of about $7 \times 7 \mathrm{~mm}$, resulting in a spatial resolution of $\sim 6.9 \mu \mathrm{m} /$ pixel. The laser sheet is generated by a $150 \mathrm{~mW}$ diode laser (Laseris Inc., Canada) making use of a light sheet optic. Due to the lighting and flow conditions (driven flow is typically $<<1 \mathrm{~mm} / \mathrm{s}$ for the given power range), a pulsed illumination is not required. Rather, consecutive images are recorded with an exposure time of 1/15 s each.

For the present setup, imaging is obtained in a horizontal plane cutting through the inflated membrane at approximately its equator, corresponding to the $z \approx 0$ plane see Fig. 1(c)). Due to (i) the finite thickness of the membrane, (ii) its curvature upon inflation into a spherical cavity, as well (iii) the fact that the material is not perfectly transparent, light sheets cutting horizontally through the cavity at heights different from $z \approx 0$ result in strong scattering effects compromising flow visualization. Similarly, a light sheet cutting vertically through the inflated hemisphere $(y=0$ plane) results in strong scattering, limiting hence our measurements to the horizontal plane $(z \approx 0)$.

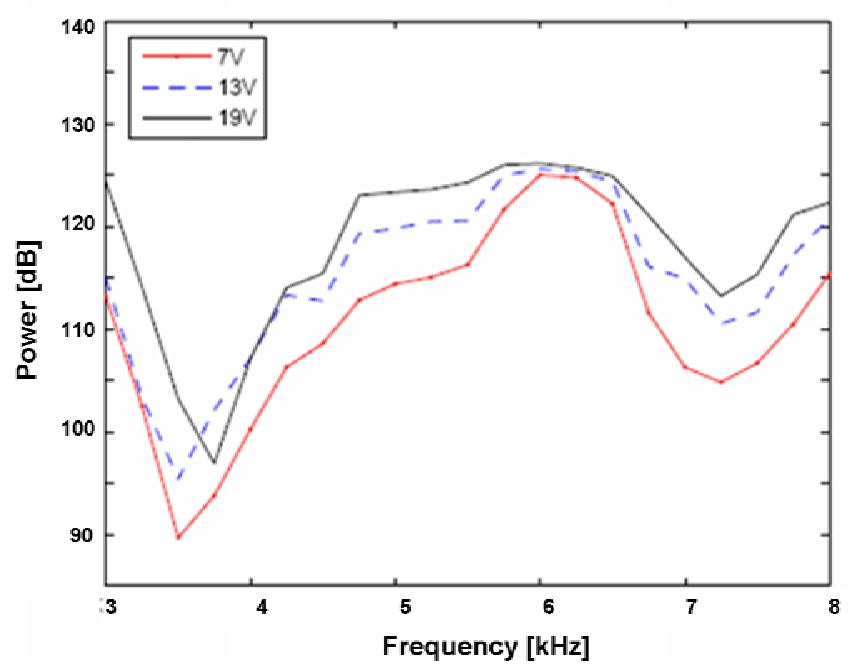

Fig. 2. Acoustic power delivered by loudspeaker vs. excitation frequency for voltage amplitudes of 7,13 and $19 V_{p p}$. Each curve results from the averaging of three independent measurements and displays the main range of frequencies investigated. 


\subsection{Analysis}

Different solid particle seeding schemes were attempted, including smoke, talc (hydrated magnesium silicate) and titanium dioxide particles. However, the deposition of solid particles on the transparent membrane quickly resulted in significant optical obstruction to the measurement plane. Different liquid solutions were tested as well and the best results for flow visualization were obtained with olive oil liquid droplets generated using an airbrush gun.

The syringe barrel is filled with air seeded with liquid droplets such that the cavity flow is effectively seeded. Two-dimensional (2D) vector displacements are obtained with a custom PIV algorithm based on cross-correlation pattern matching with sub-pixel interpolation (Roesgen, 2003). To reduce the noise level present in the raw data, while preserving detail in the image, an edge preserving median filter with a window size of $3 \times 3$ pixels is first applied to each recorded frame prior to the correlation algorithm. Cross-correlations were obtained with an interrogation window size of $128 \times 128$ pixels, with a maximum window search size in $x$ and $y$ direction of $30 \times 30$ pixels and a window shift of $16 \times 16$ pixels. Typical measurements consist in the acquisition of 100 consecutive frames, representing a physical time of $\sim 6.67 \mathrm{~s}$. Velocity vectors obtained from independent image pairs are ensemble averaged and resulting maps consist of $\sim 2100$ vectors.

Finally, pathline visualizations (i.e., streaks) are obtained by displaying the absolute value of the composite image resulting from the integration of the absolute value of the images and the subtraction of the average image to reduce the background noise.
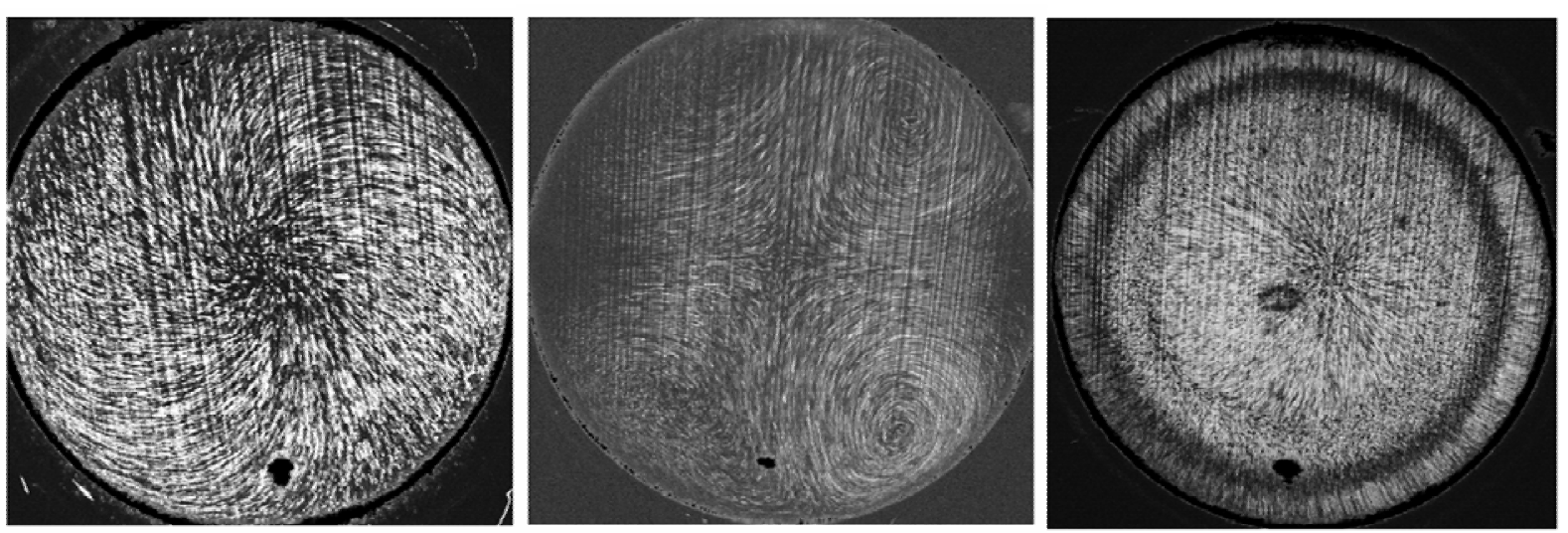

Fig. 3. From left to right, pathline visualizations of steady streaming flow at $M=120,178$, and 287 .

\section{Results and Discussion}

\subsection{Pathline Visualization}

For the range of excitation frequencies, $f$, investigated, we found several well-defined internal steady streaming flows. Examples of pathline visualizations (i.e., streaks) of some of the flows found are illustrated in Fig. 3. Such flow fields may be categorized using a dimensionless frequency parameter, $M=d(\omega / v)^{1 / 2}$ (Zhao et al., 1999), where $d$ is the characteristic diameter of the hemispherical cavity, $\omega=2 \pi f$ the angular frequency of oscillation, and $v$ the kinematic viscosity of air. $M$ may be interpreted as the ratio of a body length scale to a viscous length scale. For measurements between $M \approx 120$ - 306, the generated flows are steady and reproducible.

Qualitatively, streaming flows show strong evidence of three-dimensionality. Recently, it has been shown that such $2 \mathrm{D}$ flows measured in the horizontal plane correspond closely to solutions of the general 3D equations of creeping fluid motion inside a sphere (Sznitman and Roesgen, 2008). Evidence of such three-dimensionality is particularly noticeable at $M=120$ (Fig. 3) where a nearly axisymmetric spiraling flow with a source or sink (direction yet to be determined) is present at the center of the plane. The source/sink suggests that fluid is leaving the measurement plane and flowing in the out-of-plane $z$ axis of the cavity, while the flow remains bounded at the membrane 
interface such that mass conservation applies inside the cavity. This is also true at $M=178$ (Fig. 3), where the flow is again axisymmetric in the measurement plane and consists of two distinct regions: (i) a thin outer region near the membrane wall, separated (i.e., dark ring with no flow) from (ii) a central region with a radially oriented in- or outward flow (direction to be determined). Here flow is leaving/entering the measurement plane both at the center of the cavity $(x \approx 0, y \approx 0)$ as well as in the region described by the dark ring, suggesting again the inherent threedimensionality of the flow field. PIV analysis (following section) will help elucidate some of these flow features, and in particular the direction of the flow.
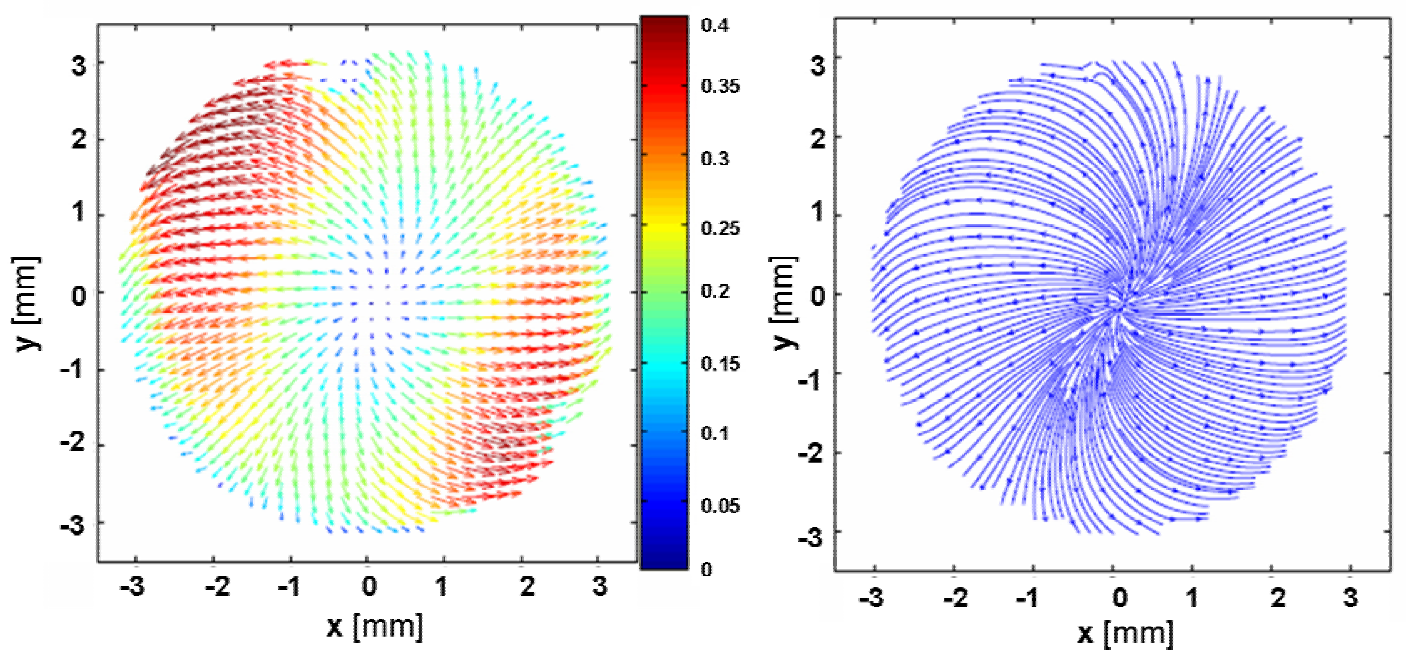

Fig. 4. (left) Ensemble averaged PIV vector plot and (right) reconstructed streamlines for an elastic membrane at $M=134\left(V_{p p}=19.7 \mathrm{~V}\right)$. Scale in $\mathrm{mm} / \mathrm{s}$ (left).

Figure 3 at $M=287$ reveals the existence of internal circulation in the shape of four vortices. Experimentally, one observes here a slight asymmetry in the generated vortices. In fact, generally, we have observed a degree of asymmetry in several of the internal streaming flows (see PIV vector maps), obtained at different values of $M$. While flow patterns are steady, the asymmetry persists over time. We suspect this may result from the fact that the inflated elastic membranes are not perfectly axisymmetric in nature. Indeed, local differences in the tension present in the membrane wall may exist when inflated at the orifice opening. Furthermore, in contrast to theoretical studies describing streaming in and/or outside spherical bodies solid spheres or gas bubbles (Wu and Du, 1997; Zhao et al., 1999), these latter geometries are perfect spheres illustrating axisymmetry in all spatial directions, while the present cavities are, rather, spherical caps or hemispheres, illustrating at best axisymmetry along the out-of-plane $z$ axis only. Thus, we expect flow patterns to be influenced by such geometrical differences.

\subsection{Velocity Fields}

Figures 4-6 illustrate PIV vector maps and resulting streamlines of the flow field for a range of dimensionless frequency values $M$. Quantitative results are obtained after ensemble averaging over 50 image pairs. As a side remark, one may note a slight distortion in some of the resulting flow fields (and consequently in the reconstructed streamlines) obtained in the region of the measurement plane nearest the laser light source $(x \approx 0, y \approx d / 2)$. This problem arises from slight scattering effects on the membrane surface, which as mentioned previously is both curved and not perfectly transparent.

Generally, we observed a rich diversity in the steady streaming flows generated inside the elastic cavity, as seen in the pathline visualizations. From the PIV map and the resulting streamlines, it now becomes clear that the flow at $M=134$ (Fig. 4) is moving radially from the center of the measurement plane towards the membrane wall, with a swirling motion. The general 
topology of the flow observable in the measurement plane bears strong qualitative resemblance with a potential flow resulting from the superposition of a counter-clockwise vortex flow with a source flow. At $M=200$ (Fig. 7), the flow looks strikingly similar to that observed at $M=134$, but here the direction of motion has changed. Here, fluid travels radially inwards, again with a slight swirling motion, while the location of the two-dimensional sink is slightly off-center. While it has been recently shown that both flows at $M=134$ and 200 may be captured by solutions of the creeping equations for fluid motions inside a sphere (Sznitman and Roesgen, 2008), it remains however unclear what precisely drives the flow to reverse its direction of motion.
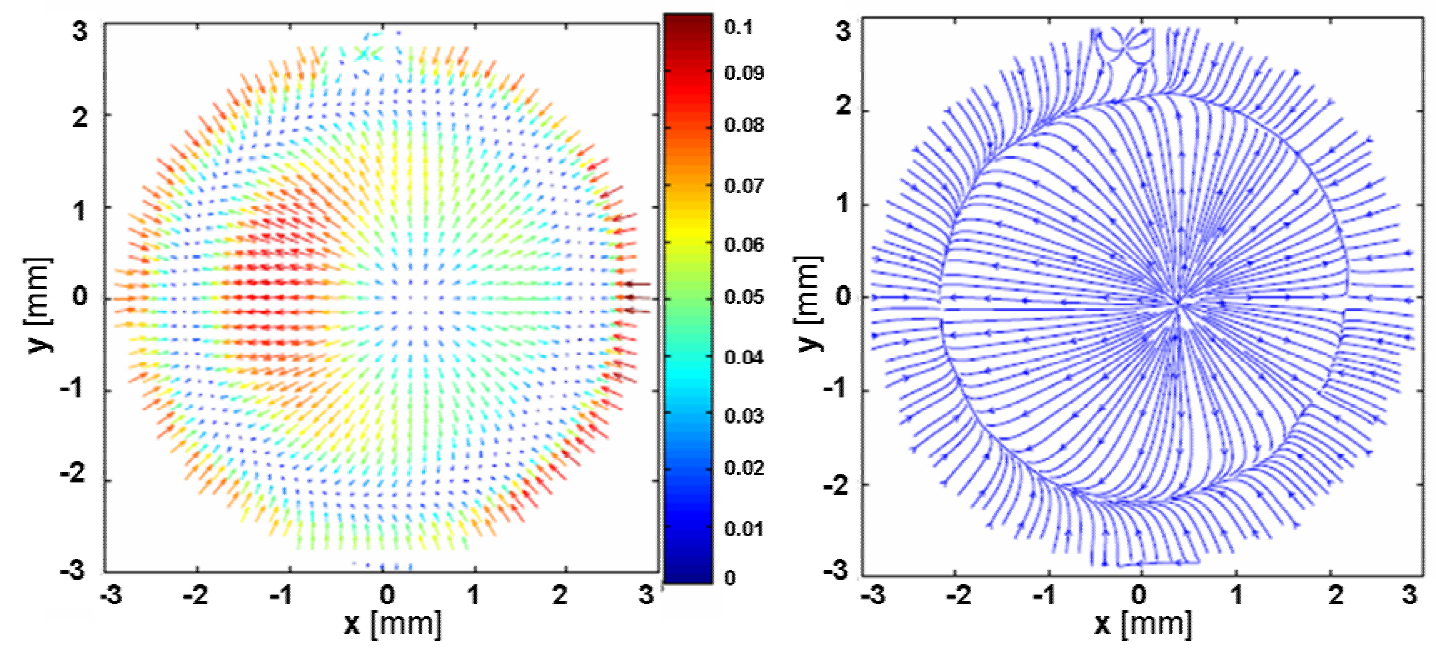

Fig. 5. (left) Ensemble averaged PIV vector plot and (right) reconstructed streamlines for an elastic membrane at $M=180\left(V_{p p}=19.1 \mathrm{~V}\right)$. Scale in $\mathrm{mm} / \mathrm{s}$ (left).

Meanwhile, at $M=180$ (Fig. 5), fluid flows towards the discernable central ring, originating both from the center of the cavity where it moves radially outwards and from the outer wall where it flows inwards towards the ring. Here again, the source-like flow in the central region is slightly offcenter, which may result amongst other from slight asymmetry in the cavity shape, as mentioned earlier. At $M=193$ (Fig. 6), we discover what appears to be a rather complex flow topology resembling the superposition of a source and a sink bounded by the circular membrane. The flow here is largely symmetrical both along the $y=0^{-}$and $x=0$-axes. Finally, at $M=306$ (Fig. 8), we observed internal recirculation made of four characteristic vortices, where streamsurfaces form a nested family of tori and a circle of elliptic fixed (stagnation) points lies at the center of each of the fours vortices nest.

For the range of power investigated, $V_{p p}=3-20 \mathrm{~V}$, flows in the plane were found to be $<1$ $\mathrm{mm} / \mathrm{s}$. Reynolds numbers are then characterized by $R e=d U / v<1$, where $U$ is taken as the mean flow velocity in the measurement plane obtained from the ensemble averaged PIV vector maps. For several streaming flow fields, we investigated the relationship between the mean flow, $U$, and the acoustic power delivered by the loudspeaker. Results are shown in Fig. 9. We found that the acoustic power followed a nearly logarithmic profile as a function of the applied voltage (Fig. 9(a)). This is particularly true for frequencies where the power was found to be the lowest (i.e., $3.3 \mathrm{kHz}$ ). Fitting the data with a curve of the form $P=C_{1} \ln \left(V_{p p}\right)+C_{2}$, where $P$ is the acoustic power (dB) and $C_{1}$ and $C_{2}$ are fitting constants, yields $\mathrm{R}^{2}>0.99$. For increasing power levels, however, the curves level off. Curves for $P$ vs. $V_{p p}$ follow the loudspeaker characteristics illustrated earlier in Fig. 2. 

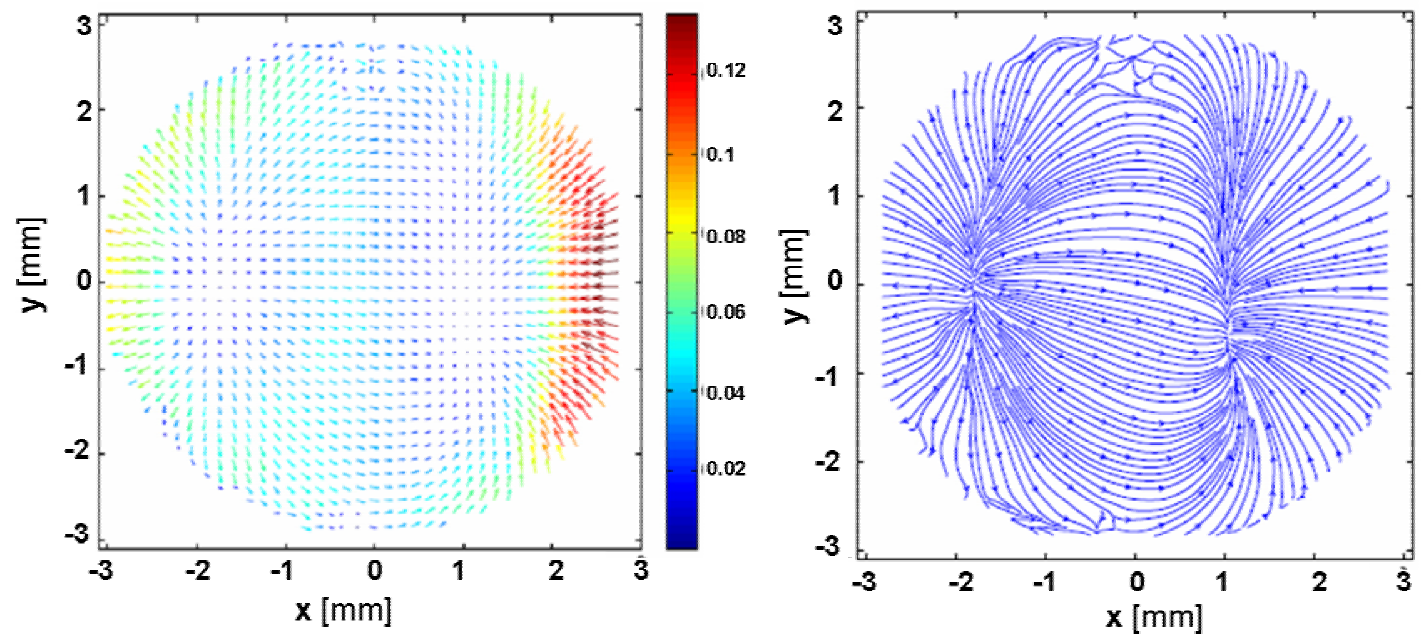

Fig. 6. (left) Ensemble averaged PIV vector plot and (right) reconstructed streamlines for an elastic membrane at $M=193\left(V_{p p}=16 \mathrm{~V}\right)$. Scale in $\mathrm{mm} / \mathrm{s}$ (left).

Correspondingly, results (see Fig. 9(b)) for the mean flow magnitude, $U$, suggest that $U$ follows approximately an exponential growth as a function of the delivered acoustic power, such that $U=$ $C_{1} e^{P}+C_{2}$, (note that the fitting constants, $C_{1}$ and $C_{2}$, are distinct from those used above in the expression $P=C_{1} \ln \left(V_{p p}\right)+C_{2}$ ). Hence, at a given value of $M$, the magnitude of the mean flow increases as the delivered power, $P$, is increased. However, the nature of the flow patterns remains unchanged (i.e., steady).
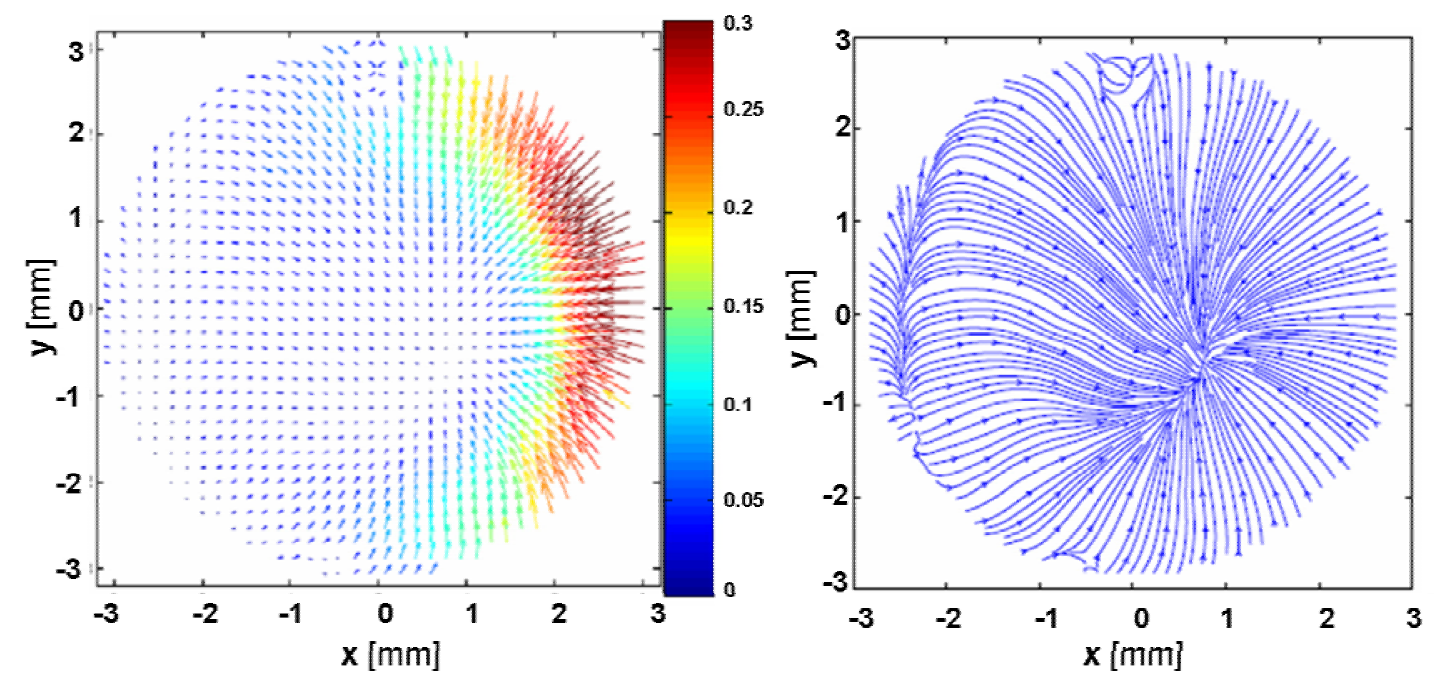

Fig. 7. (left) Ensemble averaged PIV vector plot and (right) reconstructed streamlines for an elastic membrane at $M=200\left(V_{p p}=19.4 \mathrm{~V}\right)$. Scale in $\mathrm{mm} / \mathrm{s}$ (left).

It is interesting to note that the velocity magnitude is found to be largest for streaming flows occurring at the lowest excitation frequency (i.e., $3.3 \mathrm{kHz}$, Fig. 9(b)). As the excitation frequency is increased, the magnitude of the streaming flows decreases (Fig. 9(b)). This trend follows qualitatively well with theoretical modeling of acoustic streaming, where the streaming velocity is proportional to $\omega^{-1}$ (Nyborg, 1958; Lee and Wang, 1990), such that the amplitude of resonance oscillations decreases with increasing frequency. 

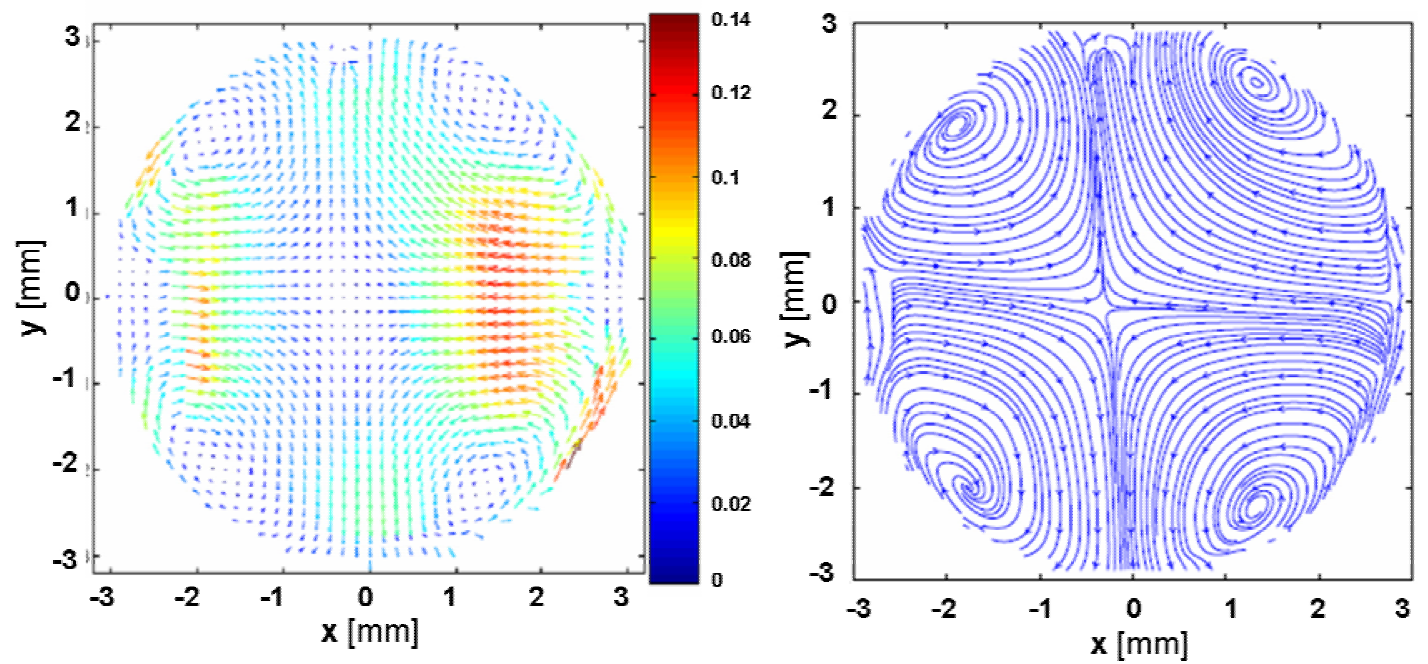

Fig. 8. (left) Ensemble averaged PIV vector plot and (right) reconstructed streamlines for an elastic membrane at $M=306\left(V_{p p}=7 \mathrm{~V}\right)$. Scale in $\mathrm{mm} / \mathrm{s}$ (left).

\section{Conclusion}

Experimental visualizations of streaming flows were obtained inside a thin elastic cavity placed in an acoustic sound field. We observed well-defined steady flow fields, generated at different values of the dimensionless frequency parameter $M$. Under the conditions tested, streaming flows exhibited mean velocity magnitudes $(<1 \mathrm{~mm} / \mathrm{s})$ which increased non-linearly with the applied acoustic power. The resulting flows suggest that fluid motion inside the cavity is inherently three-dimensional, due to the existence of sources/sinks in the computed two-dimensional vector maps. In particular, the existence of internal streaming flows illustrating well-defined recirculating vortices may be useful in the mixing of fluids on small scales and may potentially exhibit characteristics of chaotic motion, useful in the design of (micro-)mixing devices.
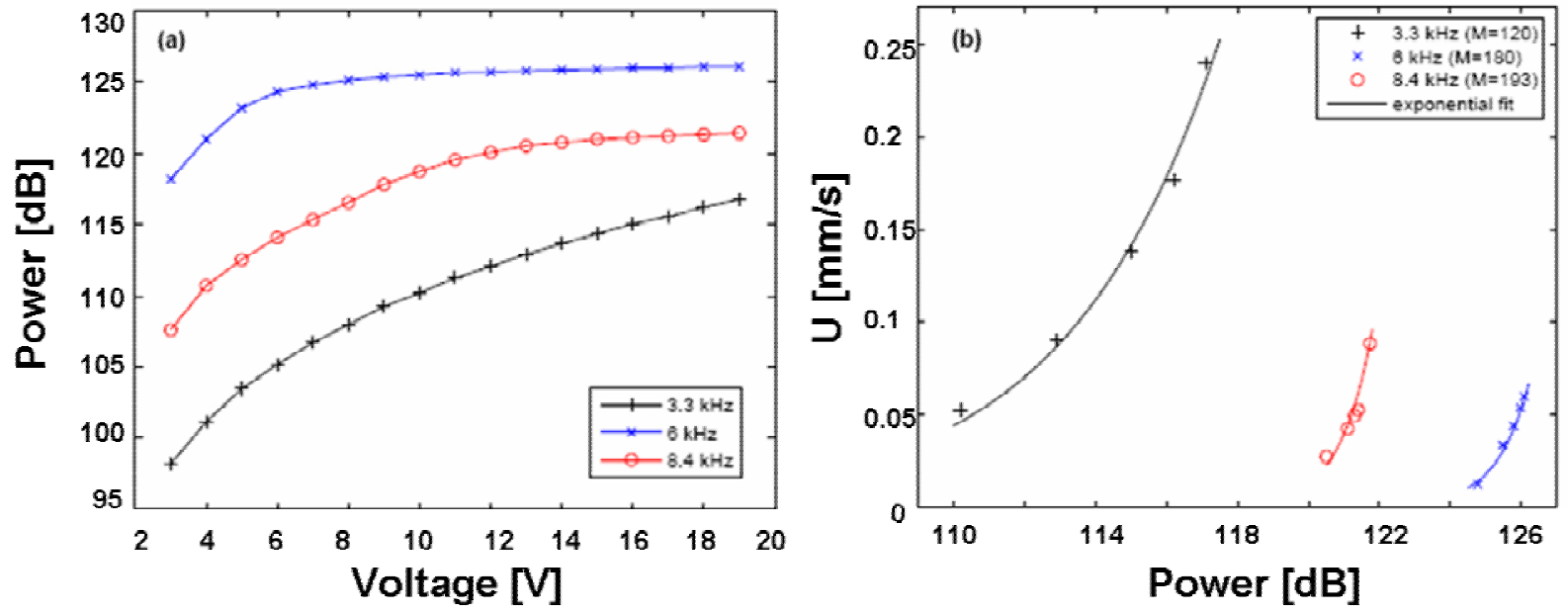

Fig. 9. (a) Loudspeaker characteristic for frequencies where streaming flows are encountered $(1.5 \mathrm{ml}$ cavity; $d \approx 6.5 \mathrm{~mm}$ ). Each curve results from the averaging of three independent measurements. Curves are of the form $P=C_{1} \ln \left(V_{p p}\right)+C_{2}$. (b) Mean streaming flow vs. loudspeaker power. Data is illustrated with exponential fits of the form $U=C_{1} e^{P}+C_{2}$. 


\section{References}

Carlsson, F., Sen, M. and Löfdahl, L., Steady streaming due to vibrating walls, Phys. Fluids, 16 (2004), 1822-1825. Davidson, B. J. and Riley, N., Cavitation microstreaming, J. Sound Vib., 15 (1971), 217-233.

Gormley, G. and Wu, J., Observation of acoustic streaming near Albunex spheres. J Acoust. Soc. Am., 104 (1998), 3115-3118. Kotas, C. W., Yoda, M. and Rogers, P. H., Visualization of steady streaming near oscillating spheroids, Exp. Fluids, 42 (2007), $111-121$.

Kolb, J. and Nyborg, W. L., Small-scale acoustic streaming in liquids, J. Acoust. Soc. Am., 28 (1956), 1237-1242.

Lee, C. P. and Wang, T. G., Outer acoustic streaming, J. Acoust. Soc. Am., 88 (1990), 2367-2375.

Lighthill, J., Acoustic streaming, J. Sound Vib., 61 (1978), 391-418.

Liu, R. H., Yang, J., Pindera, M. Z., Athavale, M. and Grodzinski, P. Bubble-induced acoustic microstreaming, Lab Chip, 2 (2002), 151-167.

Marmottant, P. and Hilgenfeldt, S., A bubble-driven microfluidic transport element for bioengineering, Proc. Natl. Acad. Sci. USA, 101 (2004), 9523-9527.

Nyborg, W. L., Acoustic streaming near a boundary, J. Acoust. Soc. Am., 27 (1958), 329-339.

Oh, Y. K. and Yang, H. D., A numerical study on the enhancement of heat transfer by pressure distributions in acoustic fields, J. Visualization, 8 (2005), 73.

Riley, N., Steady streaming, Annu. Rev. Fluid Mech., 33 (2001), 43-65.

Roesgen, T., Optimal subpixel interpolation in particle image velocimetry, Exp. Fluids, 35 (2003), 252-256.

Sznitman, J., and Roesgen, T., Acoustic streaming flows in a cavity: an illustration of small-scale inviscid flow, Physica D, 237 (2008), 2240-2246.

Tho, P., Manasseh, R. and Ooi, A., Cavitation microstreaming patterns in single and multiple bubble systems, J. Fluid Mech. 576 (2007), 191-233.

$\mathrm{Wu}$, J. and Du, G., Streaming generated by a bubble in an ultrasound field, J. Acoust. Soc. Am., 101 (1997), 1899-1907.

Wu, J., Ross, J. P. and Chiu, J. F., Reparable sonoporation generated by microstreaming, J. Acoust. Soc. Am., 111 (2002), $1460-1464$.

Yi, M., Bau, H. H. and Hu, H., Peristatically induced motion in a closed cavity with two vibrating walls, Phys. Fluids, 14 (2002), 184-197.

Zhao, H, Sadhal, S. S. and Trinh, E. H., Internal circulation in a drop in an acoustic field, J. Acoust. Soc. Am., 106 (1999), 3289-3295.

\section{Author Profile}

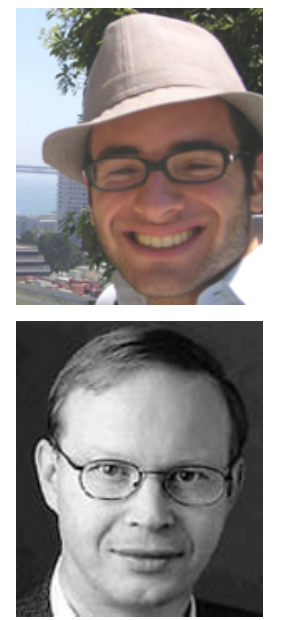

Josué Sznitman: He received his B.Sc. in mechanical engineering from MIT in 2002, M.Sc .(Dipl.-Ing.) and $\mathrm{PhD}$ in mechanical engineering from the Institute of Fluid Dynamics of the ETH Zurich (Swiss Federal Institute of Technnology) in 2003 and 2007, respectively. He is currently a postdoctoral researcher at the Department of Mechanical Engineering \& Applied Mechanics at the University of Pennsylvania. His research interests are primarily in low-Reynolds flows, biofluid dynamics and microfluidics.

Thomas Rösgen: He received his M.Sc. (Dipl.-Ing.) degree in engineering physics in 1979 at the Technical University Berlin and his $\mathrm{PhD}$ in aeronautics in 1984 from the California Institute of Technology. He is currently a professor at the Institute of Fluid Dynamics of the ETH Zurich (Swiss Federal Institute of Technology). His current research interests are centered in the field of modern electro-optical diagnostics and image processing with application to fluid dynamics. He also takes interest in problems concerning the physics of fluids and flow phenomena in constrained geometries. 